\title{
ZUM PROBLEM DES PRÜFENS UND REPETIERENS IM GEOGRAPHIEUNTERRICHT DER MITTEL- UND DER OBERSTUFE
}

\author{
Kurt Bösiger
}

Schon Becker (Lit. 3, S. 114) beklagte vor 30 Jahren die merkwürdige Erscheinung, daß die Lehrbücher der Methodik und Didaktik sich um das «Prüfen» herumdrücken und es - wenn überhaupt - verschämt «Wiederholen» nennen. Und doch greift bei der Bedeutung der Zeugnisnote gerade diese Tätigkeit des Lehrers immer noch tief in das Leben des Schülers ein. Wer dies nicht wahrhaben will, möge mit unbeteiligter Miene, aber offenen Ohren etwa den Schülergesprächen nach Schulschluß im Tram lauschen! Es ist hier nicht der Ort, über Wert und Unwert der Zeugnisse zu diskutieren; solange eine Schule Zeugnisse ausstellen muß, ist jedenfalls der Lehrer verpflichtet, den Schüler ernsthaft zu prüfen. Man könnte sich auf den Standpunkt stellen, Prüfen und Bewerten seien Gewissens- und Gefühlssache und daher nicht lernbar. Wir sind jedoch der Auffassung, daß der junge Lehrer während der methodischen Ausbildung mit den Gesichtspunkten des Prüfens und des Repetierens vertraut gemacht werden sollte - und sei es auch nur, um auf die Problematik dieser doch ausgesprochen wichtigen Lehrertätigkeit mit Hilfe praktischer Beispiele aufmerksam zu machen und den Kandidaten zum Nachdenken und zur ständigen Selbstkontrolle anzuregen. Seien wir uns bewußt, daß eine mathematisch exakte Messung der Schülerleistung - etwa gar auf Dezimalstellen - sowenig möglich ist wie ein unfehlbares Urteil des Richters oder des Arztes.

Dem Geographielehrer erwachsen aus der Eigenart und der Stoff-Fülle seines Faches noch zusätzliche Probleme. Während etwa in den Sprachfächern die wesentlichen Teile des Stoffes durch die Anwendung in Wort und Schrift ständig und automatisch wiederholt werden, geraten in der Geographie - ähnlich wie in der Geschichte behandelte Gebiete leichter in Vergessenheit. Um so wichtiger ist es für den Lehrer zu überlegen, welche Kenntnisse und Ansichten er als grundlegend betrachtet. Es sei schon jetzt unterstrichen, daß das Hauptgewicht nicht der sogenannten «Briefträgergeographie», sondern den Einsichten in die Kausalzusammenhänge zukommt. Wenn auch diese Einsichten nicht einfach «eingelernt» werden können wie das Namenwissen, so ist in den Wiederholungen - bei denen es doch um die Festigung eines echten und ganzheitlichen Bildungsgutes geht - immer wieder auf die für die Geographie typischen Wechselbeziehungen und die Wirkungen der verschiedenen Kräfte hinzuweisen (vgl. S. 101-105).

Da mit der erstmaligen Behandlung kein dauerndes Behalten des Lehrstoffes erreicht werden kann, sind Wiederholungen auch beim besten Unterricht unerläßlich. Durch Versuche hat man festgestellt, daß nach zwei Tagen durchschnittlich zwei Drittel des Gelernten vergessen sind (Lit. 6, S. 291). Daher kommt der ersten, bald durchzuführenden Repetition entscheidende Bedeutung zu; die folgenden Wiederholungen dienen nur noch der Befestigung. Sie müssen den Examencharakter verlieren und durch vorbereitete Fragen zu spannenden und mit Freude erwarteten Lektionsteilen gestaltet werden. Leider gibt es aber heute noch Lehrer, die den Wert der Repetitionen verkennen und deshalb dafür in den Schulstunden zu wenig Zeit einräumen.

Die zeitbedingte Überfülle von immer neuen, jedoch nur oberflächlich haftenden Sinneseindrücken führte zur oft bemängelten geistigen Zersplitterung unserer Gegenwart. Ein Mittel im Dienste der notwendigen Konzentration sind häufige Repetitionen - vorausgesetzt, daß der Lehrer eine klare Zielgebung seines Unterrichts vor Augen hat. Dies ist die entscheidende Bedingung zur Abklärung folgender Fragen: 
- Was soll repetiert und geprüft werden?

- Wann soll repetiert werden?

- Wie soll repetiert und geprüft werden?

\section{WAS SOLL REPETIERT UND GEPRÜFT WERDEN ?}

Der oben zitierte Geographiemethodiker BECKER (Lit. 3) unterscheidet zwischen dem in der letzten Stunde durchgearbeiteten Stoff und dem eigentlichen Wiederholungsstoff, ferner zwischen sachlichem Merkwissen und der Anwendung des Gelernten. Beim Prüfen des kürzlich durchgenommenen Unterrichtsstoffes geht es um einen Einblick in die Kenntnisse und in die Auffassung des Schülers. Es können also auch Einzelheiten gefragt werden, die bei einer spätern Prüfung oder Wiederholung wegfallen müssen. Im zweiten Fall hingegen darf nur das $W$ esentliche und das für die allgemeine Bildung Notwendige verlangt werden, also einerseits eine vernünftige Menge an gedächtnismäBig angeeignetem Namen- und Tatsachenwissen - sozusagen die vorwiegend mit Fleiß zu erwerbenden «Vokabeln» der Geographie - andererseits, als Ausdruck der Begabung, die Anwendung des Gelernten durch den Schüler. Je nach der Zielsetzung des Unterrichts kommt man zu einer Liste wesentlicher Repetitions- oder Prüfungsthemata, die nach Schulstufe, Fach und Lehrer recht unterschiedlich aussehen kann, jedoch in allen Fällen nach psychologisch-pädagogischen sowie fachmethodischen Gesichtspunkten begründet sein muß. Man vermeide jeden Schematismus: Nicht alle repetierten Stoffe brauchen geprüft zu werden; andererseits soll auch nicht jeder Prüfung eine vorbereitende Wiederholung vorausgehen! Für die Geographie der Mittel- und der Oberstufe können wir das «Repetitionsgut» etwa wie folgt gliedern:

\section{Bildungs- und V orstellungsgut}

Dem meist kleinräumig denkenden Schweizer Schüler sollen immer wieder klare Raumvorstellungen vermittelt werden (Festlandumrisse, Reliefzüge wie Gebirge, Flachländer, Hochebenen, Flußnetze, Klima- und Vegetationsgürtel, Ortslagen, Industriezonen usw.). Auch V ergleichszahlen, dosiert verwendet, schaffen Vorstellungen! Einen guten Kompromiß zwischen Zuviel und Zuwenig hat nach unserer Auffassung Annaheim mit seinen gerundeten Werten gefunden (s. S. 103). Die Gymnasiasten kennen bei wiederholter Verwendung diese «eisernen Werte» bald auswendig und können damit während mehrerer Schuljahre nützliche Vergleiche anstellen. Als Leitmotiv gelte: wenig Zahlen, diese aber häufig verwenden und stets mit Bezug auf die heimatlichen Verhältnisse.

Über den Bildungsschatz haben wir uns an anderer Stelle kurz geäußert (Lit. 5). Ein zeitgemäßer Geographieunterricht fördert neben der Fachschulung auch die allgemeine Bildung und klärt die engen Wechselbeziehungen zwischen Mensch und Natur. Da der Förderung des Bildungs- und Vorstellungsschatzes unseres Erachtens das Hauptgewicht gebührt, wurden hier bewußt die meisten Beispiele aufgeführt (s.S.101 ff). Die Aufgaben und Fragen der ersten Gruppe bergen neben materialen auch formale Bildungswerte, indem erkennendes Denken und Forschen, praktisch-wirtschaftliches Denken und Arbeiten sowie soziale, staatsbürgerkundliche, ja ästhetische Gesichtspunkte mitberücksichtigt werden.

\section{Geographische Grundbegriffe}

Sie sind wegen der unklaren oder sprachlich schlechten Formulierungen eine Quelle ewiger Mißverständnisse.

\section{A usgewählte Landschaften}

Exemplarischer Unterricht bedeutet nach unserer Ansicht Arbeit nach der Schwerpunktmethode (Lit. 4). Der viel geforderte Stoffabbau wird so zum Stoffumbau oder Stoffaufbau. 


\section{Das Nameninventar}

Über den Umfang des wissenswerten Namengutes wird man auch innerhalb der gleichen Schulstufe und innerhalb desselben Schultyps in guten Treuen diskutieren können. Sicher ist, daß sich der moderne Geographieunterricht einerseits nicht auf reine «Briefträgergeographie» beschränken darf, andererseits aber die Bedeutung eines Grundschatzes an Namen nicht verkannt werden soll. Geographie ohne Namenkenntnis ist genauso undenkbar wie ein Fremdsprachenunterricht ohne Vokabelschatz. Eine im einzelnen diskutable, im ganzen aber vertretbare Lösung geben Meriax und NigG (Lit. 10); es handelt sich dabei um ein Namenverzeichnis, das aus den praktischen Erfahrungen mit Westermanns Umriß-Stempeln herausgewachsen ist. Selbstverständlich ist auch hier nicht das Namenwissen an sich entscheidend, sondern die Beziehung der Ortsbezeichnungen in den entsprechenden Raumvorstellungen.

\section{WANN SOLL REPETIERT WERDEN :}

Huber (Lit. 8) unterscheidet folgende Formen der Wiederholung:

- die unmittelbare Wiederholung: nach jedem Abschnitt, nach der ganzen Behandlung oder nach einem größern Stoffganzen;

- die periodische Wiedcrholung: von Zeit zu Zeit, womöglich nach neuen Gesichtspunkten, z.B. durch Vergleiche, andere Stoffgruppierung (etwa Faltengebirge, Landschaftsgürtel, Ballungszentren der Menschheit, Kornkammern der Erde, Kohlenregionen, Industrieräume 「es gilt hier der Satz: «Nur was oft wiederholt wird, haftet!»]);

- gelegentliche Wiederholungen: sie ergeben sich aus dem Unterrichtsablauf. Man kommt auf behandelte Themata zurück, sei es innerhalb des Faches oder als Querverbindung von Fach zu Fach. Beispiele:

Geographie: Mittelmeerländer

Geographie: Deutschland

Geographie: Großbritannien

Geographie: Afrika
- Geschichte: Römerreich

- Geschichte: Glaubensspaltung

- Geschichte: Kolonisation der Erde

- Geschichte: Entdeckung und Erschließung

Selbstverständlich sind die Wiederholungen vernünftig und planmäßig zu verteilen. Insbesondere ist eine Häufung gegen Ende der Zeugnisperiode zu vermeiden. «Kleine Portionen, häufig und gut verteilt in der Wiederholung, bringen ein besseres Resultat zustande als große Quantitäten mit wenig Wiederholungen oder als kleinste Teile in allzu häufiger Abfolge.»(Lit. 7, S. 35). Meumann (Vorlesungen Bd.2, S. 128) zog aus Versuchen den Schluß, daß die baldige erste Repetition des Gelernten der eigentliche Träger des Behaltens ist und die folgenden Wiederholungen nur noch einer letzten Befestigung dienen.

Auch wir sind der Auffassung, daß häufig, kurz und unter Verwendung des geistigen Kartenbildes wiederholt werden soll. Allerdings scheint es uns übertrieben, prinzipiell in jeder Schulstunde zu wiederholen, da bei diesem zu starren System oft der geschlossene Aufbau der Lektionen gestört wird. Neben den postulierten Kurzrepetitionen nach 2 bis 3 Lektionen können etwa alle drei Wochen ausführliche, meist 1 Lektion in Anspruch nehmende Wiederholungen eines geschlossenen Stoffgebietes in kleinerem oder größerem Umfang durchgeführt werden, namentlich vor angekündigten umfangreicheren Prüfungsarbeiten auf der Mittelstufe. Zusammenfassende Repetitionen nach Abschluß der Behandlung größerer Abschnitte dienen am besten der Schaffung eines «eisernen Wissensbestandes», sofern wirklich alle behandelten Gebiete einbezogen werden (Lit. 6, S. 291). Reicht die Zeit dazu nicht aus, so leisten Querschnittrepetitionen durch das gesamte geographische Bildungsgut wertvolle Dienste.

\section{WIE SOLL REPETIERT UND GEPR ÜFT WERDEN?}

Die sinnvolle Repetitionsübung umfaßt weit mehr als eine bloß kopiehafte Wiederholung, namentlich bei einem schon teilweise eingeprägten Lehrstoff (Lit. 7, S. 33 ff). Damit eine Repetition wirkungsvoll ist, sollen bekannte Tatsachen in einem neuen $\mathrm{Zu}$ - 
sammenhang oder in einer neuen Beleuchtung auftreten. Wie die Einführungen sollen auch die $\ddot{U}$ bungen abwechslungsreich gestaltet werden und zudem richtig dosiert sein.

Es entspricht der Vielseitigkeit des Faches, wenn die Repetitions- und Prüfungsmöglichkeiten im Geographieunterricht besonders mannigfaltig sind, so daß keinerlei Langeweile aufkommt. Die nachstehenden, zur Hauptsache aus dem Unterricht an der Kantonalen Handelsschule in Basel herausgewachsenen Beispiele erheben keinen Anspruch auf Vollständigkeit und sollen als Anregungen aus einer Fülle von Möglichkeiten aufgefaßt werden, die je nach Schulart und Schulstufe abzuwandeln sind. Wir richteten unser Augenmerk bewußt auf das Wiederholen und Prüfen in schriftlicher Form, obwohl vieles auch für den noch variantenreichern mündlichen Unterricht Geltung haben mag. Die angeführten Beispiele sind kaum für eigentliche Abschlußprüfungen verwertbar, hingegen wohl zu schriftlichen Prüfungsarbeiten oder nicht bewerteten Repetitionen. Die abwechslungsreiche Vielfalt darf selbstverständlich nicht in systemlose Spielerei ausarten, womit dem noch oft mißverstandenen Fach schlecht gedient wäre. Andererseits sind wir der Auffassung, daß sich eine «gerechte» Geographienote aus mehreren Komponenten zusammensetzen soll, können doch gerade hier - im Grenzgebiet zwischen Natur- und Geisteswissenschaften - vielerlei gute Kräfte im Schüler angesprochen werden. Es ist unseres Erachtens beispielsweise falsch, Zeugnisnoten in der Geographie einseitig zu basieren, sei es nur auf Tatsachenwissen oder nur auf Ausdrucks- und Darstellungsfähigkeiten oder nur auf kausalem Denkvermögen. Allerdings wird je nach Schulstufe und Stoffgebiet das eine oder andere mehr Gewicht erhalten. Zweifellos liegt bei der Mittelstufe das Hauptgewicht auf dem $W$ as und dem Wie, auf der Oberstufe hingegen bei der Förderung des kausalen Denkvermögens.

Nachstehend seien - entsprechend unserer Gliederung des «Repetitonsgutes» (siehe vorn) - einige Möglichkeiten angedeutet, wobei insbesondere auch die Kausalzusammenhänge zwischen Klima und Wirtschaft und die Beziehung der geographischen Namen mit den entsprechenden Raumvorstellungen betont werden. Weder unsere Gliederung des Repetitionsgutes noch die Zuweisung der Beispiele zu den vier Untergruppen ist als einzige vertretbare Lösung zu verstehen, um so weniger, als eine scharfe Abgrenzung bei der Gruppierung nicht in jedem Falle möglich ist. Hingegen hoffen wir mit diesem Versuch einen an regenden Beitrag in einem wichtigen Teilgebiet der Geographiemethodik zu liefern.

\section{Beispiele zur Förderung des Bildungs- und Vorstellungsgutes}

\section{Repetition und Prüfen mit Lichtbildern oder Diagrammen}

Schriftlich (bei Halblicht) oder mündlich, vor allem nach Typenbildern schweizerischer Landschaften.

Frageschema: 1.Was? 2. (eventuell) Wo? 3. (eventuell) Warum? Beliebt sind sogenannte «Detektivstunden»!

Leitschema für das Bild-und Kartenstudium (nach H. Annaheim, Basel)

a) Naturgrundlagen

1. Umgrenzung, 2. Lage (Gebiet), 3. Größe und Volksdichte, 4. Relief, 5. Klima a) Temperatur,

b) Niederschlag, 6. Gewässer, 7. Vegetation (und Tierwelt).

b) Mensch und Menschenwerke

8. Mensch (Rasse, Zahl, Sprache, Religion), 9. Siedlungen, 10. Wirtschaft a) Landwirtschaft,

b) Gewerbe, Industrie und Bergbau, c) Handel, 11. Verkehr, 12. Politische Verhältnisse.

c) Einteilung in verschiedene Landschaften

Westermanns Arbeits- und Lösungskarten

a) W. Tiede: Hilfen für die Arbeit am Atlas (je 32 Aufgaben und Lösungskarten). Georg Westermann Verlag 1956.

Zweck: Übungen im Gebrauch des Atlasses sowie Repetitionen.

Voraussetzung: Kenntnis von Gradnetz und Maßstab.

Themata: Hauptländer Europas (mit besonderer Berücksichtigung Deutschlands), außereuro- 
päische Erdteile, Aufgaben zur Allgemeinen Geographie.

Mcthode: Gruppen- oder Einzelarbeit, im Anschluß an Kartenlehre oder zur Einführung in die Geographie Deutschlands, insbesondere in obern Klassen der Mittelstufe oder untern Klassen der Oberstufe.

b) K. StARKE: Klimakartei. Ein Beitrag zum erdkundlichen Arbeitsunterricht. Erläuterungsheft mit vielen Anregungen und Beispielen für alle Klassenstufen. Georg Westermann Verlag 1952.

\section{Repetition und Prüfung in Aufsatz-, Stichwort-, Tabellen- und Skizzenform}

Antworten auf Teilfragcn (statt cin Gesamtthema) geben oft bessern Aufschluß über das geographische Denkvermögen und die Kenntnisse der Schüler und sind zudem leichter zu bewerten (eventuell wichtigere Fragen doppelt bewerten).

a) Bcispiel zu: Vereinigte Staaten von Nordamerika (verschiedene Aufsatzformen):

1. Landschaftsbilder aus dem Yellowstone-Nationalpark (Beschreibung), 2. Die Trockenlandschaften im W der USA (Typenschilderung), 3. Die Industrielandschaften um die Großen Seen und im E der USA - ein geographischer Vergleich (anspruchsvollere Gliederungsaufgabe).

Jede der Antworten wird mit o, $1 / 2,1,1 \frac{1}{2}$ oder 2 Punkten bewertet. Im Idealfall also $3 \times 2=$ Note 6.

b) Bcispicl zu Afrika (Aufsatz-, Stichworttabellen- und Skizzenform kombiniert):

1. Klima und Pflanzenwelt im Kongo (Aufsatz), 2. Steppe und Wüste in Afrika - ein geographischer Vergleich (Tabelle), 3. Der Passat (Erklärung in Stichworten mit Skizze).

\section{Profilzeichnen}

Voraussetzung: Gründliche Besprechung mit Übungen, beispielsweise bei Frankreich.

Aufgabe: $W-E$-Profil durch dic USA:

San Francisco - Großer Salzsee - Washington

Höhenmaßstab: $500 \mathrm{~m}$ Höhe $=5 \mathrm{~mm}$ oder 1 Häuschen

Längenmaßstab und übrige Grundlagen: nach Mittelschulatlas S. 11t

Empfehlenswert sind insbesondere auch Kausalprofilc (vgl. Lit. 9).

Systematische Fragestellung in der Reihenfolge der behandelten Stoffe

Der Lehrer hat entweder die Fragen so zu formulieren, daß eine Kurzbeantwortung möglich ist, oder eine Auswahl zu treffen aus größeren Fragcnkrciscn.

a) Beispiel zur. Wirtschaftsgeographic der Schwciz

Statt Einzelfragen, die vorwiegend auf die Prüfung von Gedächtniswissen zielen, können einige systematisch geordnete Themata behandelt werden, wobei die Art der Beantwortung zur Förderung des Ausdrucksvermögens in der Muttersprache sowie zur Hebung des Darstellungsvermögens variantenreich vorgeschrieben werden kann. Jede Frage wird mit 1/4, 1/2, 3/4 oder 1 Punkt bewertet; Maximum: 5 Punkte = Note 6.

1. Die geographischen Grundlagen der schweizerischen Wirtschaft (naturbedingte Vor- und Nachteile)

2. Warum hat sich die Schweiz vom Bauernland zum Industriestaat gewandelt? (Aufsatz)

3. Unsere Bodenschätze (Tabelle: was? wo?)

4. Geographische Verbreitung und Bedeutung von Ackerbau und Graswirtschaft (Stichworte)

5. Zentren und Zweige der schweizerischen Metallindustrie (Tabelle: wo? was? oder Aufsatz)

Variante für Mädchenklassen: Zentren und Zweige der Schweiz. Bekleidungsindustrie.

b) Bcispiel Nordamerika

a. Relief

1. Der Vierklang im Relief (Hochgebirge im W - Mississippitiefland - Mittelgebirge im E - östliches Küstentiefland)

2. Der Grand Cañon (Skizze mit Erklärung, eventuell nach Lichtbild)

3. Geysire (Skizze mit Erklärung)

b. Klima und Vegetation

1. Die Landschaftsgürtel Nordamerikas (kalte - gemäßigte — subtropische Zone: Repetition der Klimatypen)

2. Klimatischer Gegensatz zwischen dem Ostrand Nordamerikas und Westeuropa (Golfstrom!)

3. Tundra, subpolarer Nadelwald und Prärie in Kanada

4. Warum ist es im Großen Becken so trocken? (starke Sonneneinstrahlung, Gebirgsrahmen)

c. Wirtschaft

1. Erscheinungen der Soil-Erosion in den USA und Maßnahmen zur Rettung des Landes

2. Phasen der wirtschaftlichen Entwicklung in Kalifornien (Viehzucht - Goldrausch Viehzucht - Bewässerungswirtschaft - Erdöl - Industrialisierung)

3. Die Landwirtschaftszonen der USA (Skizze mit Text oder Stempel) 
4. Bergbau und Industriezentren der USA (Tabelle: Ort/Bedeutung oder Stempel)

5. Die weltwirtschaftliche Bedeutung der USA (Aufsatz)

Repetitionsfragen, wie sie sich die Schüler selbst stellen

Beispiel Großbritannien. (Auswahl nach Lit. 14, S. 185)

- Welche Wirkungen übt das Meer auf Großbritannien aus? (auf Umrisse, Klima und Mensch)

- Nenne einige Inseln um England!

- Wie groß ist die längste $\mathrm{N}-\mathrm{S}$-Ausdehnung Großbritanniens?

- Warum gibt es in England wenig Wald?

- Welche Meeresteile der Ostküste entsprechen bestimmten Buchten der Westküste?

- Aus welcher Zeit stammen die englischen Gebirge, und welches Aussehen haben sie deshalb?

- Wo finden wir die meisten Kohlen?

- In welchen Städten gibt es Eisenindustrie?

Bei Wiederholungen durch Schülerfragen geht es meist recht bunt und ungeordnet zu. Aber gerade die freie Form des Selbstprüfens macht den meisten Schülern Spaß, und zudem erzieht ein gelegentliches Durcheinander der Fragen zur Geistesgegenwart.

\section{Der geographische Vergleich}

Als ausgezeichnetes Mittel zur Förderung des Verständnisses und zum Verdeutlichen geographischer Vorstellungen nennt schon P. WAGNER (Lit. 14) den Vergleich. Man vergleicht beispielsweise im Sinne zeitsparender Gesamtrepetitionen am Ende des Schuljahres Landschaften oder Länder nach Umriß, Relief, Gewässernetz, Klima und Wirtschaft oder greift cin Objekt heraus.

Beispiel a. Vergleiche Norwegen und Schweden oder Nord- und Südamerika nach dem länderkundlichen Schema (als Generalrepetition)

Beispicl b. Vergleiche die Flußsysteme des Kongos und des Amazonas (als schriftliche Einzelarbeit mit oder ohne Atlas, je nachdem, ob schon beide Kontinente behandelt wurden)

Bcispiel c. Liste von Vergleichswerten (nach H. ANNahem, Basel):

\section{VERGLEICHSWERTE}

1. Geographische Koordinaten von Basel $+71^{\circ}$ n.B., $71^{\circ}{ }^{\circ}$ o.L.

2. Strecken:

Bahnhof SBB - St. Albantor

Birsfelden - Aesch

Genf - Bodensee (Fußach)

Basel - Frankfurt a.M.

3. Flächen:

Kanton Basel-Stadt

Basel-Stadt und Basel-Land

Bodensee/Genfersee je

Uri , Thurgau je

4. Höhen:

Basel

Chur

Gempen

$\mathrm{Pa} ß w$ ang

Rigi (wie Oberengadin)

5. Klima:
a) Temperatur:
Basel Januar-Mittel
Juli-Mittel
Jahresmittel

6. Abflußmengen:

7. Areale Schweiz:
10oo-km-Strecken von Basel aus:

$1 \mathrm{~km}$ Jütland (Kopenhagen)

$10 \mathrm{~km}$ Theiss (Belgrad)

$300 \mathrm{~km}$ (S-Spitze) Sardinien

$300 \mathrm{~km}$ Brest

Rheinlänge

$1300 \mathrm{~km}$

to $\mathrm{km}^{2}$ Wallis

$5000 \mathrm{~km}^{2}$

$450 \mathrm{~km}=\quad$ Schweiz

$550 \mathrm{~km}^{2} \quad$ Frankreich

$1000 \mathrm{~km}^{2}$ Europa

Grönland

$41000 \mathrm{~km}^{2}$

$550000 \mathrm{~km}^{2}$

1o Mio km2

$2 \mathrm{Mio} \mathrm{km}^{2}$

$2100 \mathrm{~m}$

$4550 \mathrm{~m}$

$463+\mathrm{m}$

$\begin{array}{lll}1200 \mathrm{~m} & \text { Mt. Blanc } & 4800 \mathrm{~m} \\ 1800 \mathrm{~m} & \text { Gotthardpaß } & 2100 \mathrm{~m}\end{array}$

$\begin{array}{lll}1200 \mathrm{~m} & \text { Mt. Blanc } & 4800 \mathrm{~m} \\ 1800 \mathrm{~m} & \text { Gotthardpaß } & 2100 \mathrm{~m}\end{array}$

\begin{tabular}{|c|c|c|}
\hline 0 & Basel & $80 \mathrm{~cm}$ \\
\hline $19^{\circ}$ & Staldenried (bei Visp) & $53 \mathrm{~cm}$ \\
\hline $10^{\circ}$ & Mönchsgrat & $41+\mathrm{cm}$ \\
\hline & $\begin{array}{l}\text { Rhein, Mittel } \\
\text { Birs, Mittel } \\
\text { St.-Alban-Teich, Mittel }\end{array}$ & $\begin{array}{r}1000 \mathrm{~m}^{3} \text { Sek. } \\
10-90 \mathrm{~m}^{3} \text { Sek. } \\
7 \mathrm{~m}^{3} \text { Sek. }\end{array}$ \\
\hline $\begin{array}{l}\text { nprod } \\
\text { ald } \\
\text { ckerba }\end{array}$ & Wiesen, Weiden & $\begin{array}{l}22 \% \\
2+\% \\
54 \%\end{array}$ \\
\hline
\end{tabular}


8. Volksdichte:

Schweiz

Basel-Land

9. Höhengrenzen:

Waldgrenze

gi

Oberengadin/Wallis
Europa

13n Graubünden

350 Afrika

$1800 \mathrm{~m}$ Schneegrenze Säntis

$2300 \mathrm{~m}$

\section{Beispiele zur Erarbeitung oder Festigung geographischer Grundbegriffe}

Arbeiten mit Atlas (Karte) und Lehrbuch

Um den Schülern zu zeigen, daß sich echte Geographie keineswegs im Auswendiglernen von «Briefträgerwissen»erschöpft, sind gelegentliche Übungen zum vernünftigen Gebrauch unserer obligatorischen Lehrmittel recht dankbar. Man staunt immer wieder, wie unpraktisch und wenig exakt viele Schüler die einfachsten Aufgaben angehen.

Beispiele:

1. Bestimme die äußersten Punkte der Schweiz im Gradnetz (Schülerkarte)

2. Bestimme die genaue Lage der City Londons (Atlas-Spezialkarte)

3. Welche Stadt liegt auf $13 \frac{1}{\circ} 2^{\circ}$ o.L. und $521^{\circ}$ n. Br.? (Berlin)

4. Welches ist der größte, welches der volksreichste Staat Europas? (Lehrbuch, statistischer Teil: Europäisch-Rußland)

5. Welches europäische Land weist die größte Volksdichte, welches hingegen die geringste Bevölkerungsanzahl auf? (Lehrbuch, statistischer Teil: Belgien bzw. Vatikanstaat)

Vergleiche mit den schweizerischen Verhältnissen

6. Miß die N-S-Ausdehnung Afrikas (Luftlinie) auf dem $20^{\circ}$ ö.L. (ca. $7500 \mathrm{~km}$ ) und schätze die Größe des Viktoriasees ( $66250 \mathrm{~km}^{2}$ ) im Vergleich zur Schweiz.

Definiere folgende geographische Begriffe

Beispiele:

1. Unterschied zwischen Falten- und Rumpfgebirge

2. Unterschiede zwischen feuchten, wechselfeuchten, trockenen Tropen

3. Unterschied zwischen Luv und Lee

4. Urproduktion

\section{Amerikanisches Testsystem}

Nur als Abwechslung anzuwenden, da die Methode - mit einer «Spekulationschance» bei Nichtwissen - zwar anregend, aber auch fragwürdig ist.

Beispiele zur Allgemeinen Geographie

Der Lehrer gibt etwa zwanzig richtige und falsche Definitionen allgemeingeographischer $B C$ griffe. Der Schüler hat zu überlegen, ob die Erklärung mit $\mathrm{r}$ (= richtig) oder $\mathrm{f}$ (= falsch) zu bezeichnen ist, etwa so:

1. «Schelf»= der von der Flachsee (bis $200 \mathrm{~m}$ Tiefe) bedeckte Küstensockel (richtig)

2. «Lagune» = deutsche Bezeichnung für den italienischen Begriff «Haff» (durch Nehrung vom

Meer abgetrennte seichte Meeresbucht oder Strandsee) (falsch; richtige Lösung umgekehrt)

\section{Beweise folgende Sätze}

1. Asien ist reich an Gegensätzen

2. Die Schweiz ist ein Alpenland

\section{Unterhaltsame Prüfungen}

«Rätsel zur Schweizer Geographie»(Lit. 13, März 1960).

«Wo liegt das?» (Lit. 13, November 1960).

«Die Bodengestalt der Schweiz» (Lit. 13, Dezember 1961).

«Geographie in der Küche»(Lit. 13, Januar 1962).

\section{Beispiele zur Behandlung ausgewählter Landschaften}

\section{Repetition in Form einer Reise}

Man läßt die Schüler von $A$ nach $B$ reisen und alle gesehenen geographischen Tatsachen mitteilen. Bei dieser Repetitionsart kommt die Vielseitigkeit der Geographie zur Geltung, da die An- 
wendung von Kenntnissen aus den verschiedenen Gebieten verlangt wird; sie regt das Interesse der Schüler an und eignet sich besonders zur Wiederholung größerer Gebiete.

Beispicle:

a) Fahrt mit dem Schiff der europäischen Küste entlang und berichtet über die Küstenstädte

b) Zählt Gebirgszüge, Nebenflüsse, Landschaften und Städte am Rhein auf (von der Quelle bis zur Mündung)

c) Was siehst du auf einer Eisenbahnfahrt von Basel nach Chiasso? (Gotthardlinie)

Zusammenfassende Repetition oder Prüfung eines größeren Stoffgebietes

Brispiel Afrika (s. Lit. 13, November/Dezember 19+6)

1. Gründe für die späte Erschließung (klare Herausarbeitung der einzelnen Tatsachen in Aufsatz-oder Stichwortform verlangen!)

2. Einzeichnen der wichtigsten Bodenformen in Umriß-Stempel (auch in Tabcllonform möglich)

3. Landschaftliche Gliederung

4. Einzeichnen der noch existierenden Kolonialgebiete in Umriß-Stempel

5. Repetition des Namenwissens: nach Wahl des Schülers: physische oder politische Karte Afrikas (Westermann-Stempel oder eigener Umriß)

6. Wirtschaftskarte: nur die Anfangsbuchstaben bestimmter Produkte in einer der Bedeutung entsprechenden Größe einzeichnen lassen

\section{Leistungsvergleiche}

a) Selbständige Einzelarbeit: Südancrika (Analyse und Leistungsvergleich), vgl. Lit. 11, S. $213 \mathrm{f}$.

b) Repctitionstabelle zu Südamerika. Die Schüler haben folgende Kolonnen auszufüllen: Land (alphabetisch) — Hauptstadt — bedeutende Häfen — wichtige Ausfuhrprodukte

c) Arbeits- und Leistungsvergleich: Australien, vgl. Lit. 11, S. $22+\mathrm{f}$

\section{Abwechslung bei der Repetition und Festigung des Nameninventars}

\section{Fragenschema zur Generalrepetition der Schweiz}

Generalrepetitionen über ein größeres Stoffgebiet geben Aufschluß darüber, ob das Grundwissen noch vorhanden ist. Der Lehrer erhält so - namentlich bei unangekündigten Arbeiten ein reales Bild über den Lehr-und Lernerfolg.

Nach dem folgenden Fragenschema kann das als notwendig erachtete Namenwissen rasch und vielseitig wiederholt werden.

1. Wo entspringen folgende Flüsse?

2. In welche Seen fließen folgende Flüsse?

3. In welches Gewässer münden die aufgeführten Flüsse?

t. Welche Orte liegen an der Mündung der aufgezählten Flüsse?

5. $\mathrm{Zu}$ welcher Bergkette gehört dieser Berg?

6. Welche Bergketten begrenzen folgende Talschaften?

7. Welche Talschaften werden durch den genannten Paß verbunden?

8. Wie heißt der Paß, der nachstende Täler verknüpft?

9. Nenne die Paßfußorte im N und S folgender Pässe!

10. An welchem Gewässer liegt dieser Ort?

11. Nenne einige Schnellzugsstationen der ......-Linie!

12. Wie heißt die Hauptstadt des Kantons...?

13. Wie heißen die Orte an den Ausgängen des...-Tunnels?

1+. Wie nennt sich die Talstation der ...-Bergbahn?

\section{Wiederholungen mit Hilfe von Westermann-Umrißstempeln}

Einige der zahlreichen Möglichkeiten:

a) Repetition eines kleinen, nur überblicksweise besprochenen Landes (z. B. Belgien oder die Niederlande):

Freies Einzeichnen des Namen- und Tatsachenwissens sowie der wichtigsten Bodenschätze und Ackerbauprodukte

Sauberkeit und Übersichtlichkeit der Darstellung sind unbedingt in die Bewertung einzubeziehen

b) Repetition eines größern, genauer besprochenen Landes (z.B. Deutschland, Frankreich, Italien, Großbritannien) :

1. wie unter a), jedoch Land in natürlicher oder schematischer Art aufteilen (2 Abteilungen)

2. Der Lehrer grenzt thematisch ab, was eingezeichnet werden soll (z.B. Gebirge und Gewässer oder Stätte und Verkehrslinien) 
3. Bestimmte (numerierte) Lehrerfragen zum Namenwissen oder zur Wirtschaft (eventuell gemischt), entweder systcmatisch gcordnct (leichter) oder bewußt unsystcmatisch gestellt

\section{Testfragen zur "Briefträgergeographie" Europas}

nach folgender Art (vgl. S. 1ot: amerikanisches Testsystem) :

1. Die Hauptstadt Lettlands heißt: 1. Tallin, 2. Riga, 3. Kowno; richtige Lösung: 2

2. Korsika gehört zu: 1. Frankreich, 2. Spanien, 3. Italien; richtige Lösung: 1

3. Welcher der folgenden Staaten grenzt nicht an den Rhein? 1. Niederlande, 2. Frankreich, 3. Belgien; richtige Lösung: 3

4. Welche der nachstehend genannten Städte liegt nicht am Rhein? 1. Köln, 2. Karlsruhe, 3. Mainz; richtige Lösung: 2

5. Fließt die Oder in 1. die Ostsee, 2. die Nordsee, 3. den Atlantik; richtige Lösung: 1

6. Welcher der 6 Staaten grenzt nicht an die Donau? 1. Österreich, 2. Rumänien, 3. Albanien $t$. Jugoslawien, 5. Bulgarien, 6. Tschechoslowakei; richtige Lösung: 3

\section{Wiederholungsfragen}

Vgl. Lit. 13, März 1961: Empfehlung, einen Zcttclkatalog mit Fragekarten anzulegen, die zugleich zum Denken anregen, die Ausdrucksfähigkeit fördern, Abwechslung bieten und Querverbindungen zu andern Fächern schaffen.

Beispicle:

- Welche Kantone grenzen nicht ans Ausland?

- Zeige Morgarten, die Schöllenen, Zermatt usw. auf der Karte und erzähle dazu!

- Du planst eine mehrtätige Velotour in den Jura. Welche Wege wählst du? Welche Sehenswürdigkeiten merkst du dir?

- Auf welcher Rotite erreicht ein Bündner Parlamentarier aus dem Misox im Winter seine Kantonshauptstadt?

\section{Wiederholungsaufgaben}

Vgl. Lit. 13, November 1960: Anregende Beispiele von Skizzen (in verschiedenen Himmelsrichtungen angefertigt): Pässe, Talausgänge, Eisenbahnknotenpunkte und -Endstationen, Flußmündungen in Seen, Badeorte, Brückenstädte, Aussichtspunkte usw.

\section{Gute Hilfsmittel zur Repetition der "Briefträgergeographie"}

a) Repetitionskarten von WALTER ANGST Bczug: Verlag der Sekundarlehrerkonferenz des Kt. Zürich, Loorstraße 1t, Winterthur. Zeitsparende und abwechslungsreiche Anwendungsmöglichkeiten zur Wiederholung und Vertiefung des Tatsachenwissens sind in den Begleitworten zu den Wiederholungskarten angegeben.

b) Repetitionen mit Umriß-Stempeln

Mit Hilfe von Westermann-Umriß-Stempeln können die Wiederholungen den individuellen Bedürfnissen angepaßt werden.

Bcispicle (auf Weltstempel): Einzeichnen der Weltverkehrslinien und Großhäfen, der Großstädte, Landschaften und weltwirtschaftlich wichtigen Produkte auf ca. $60^{\circ}, 50^{\circ}$ und to nördlicher Breite.

c) Länderumrisse

Nach Atlaskarten können in verschiedenen Maßstäben Länderumrisse als Deckpausen hergestellt werden (ohne jeden Karteninhalt). Die Schüler notieren in Wcttbowcrbsform den Namen des Landes, das von den einzelnen numerierten Umrissen dargestellt wird.

\section{LITERATURHINWEISE}

Aus der großen Auswahl an methodischer und schulgeographischer Literatur, die eine Fülle von weiteren Anregungen und Repetitionsmöglichkeiten aufzeigen, seien hier nur die zitierten ältern Werke sowie einige neuere Arbeiten herausgegriffen:

1. Annaherm H.: Bedeutung und Möglichkeiten der Landschaftskunde im Geographieunterricht. "Gymnasium Helveticum»1960/61, Nr. 4. H. R. Sauerländer, Aarau. - 2. Bauer L.: Erdkundliches Lehrwerk für höhere Lehranstalten. Verschiedene Bände. R. Oldenbourg München. - 3. BECKER A.: Methodik des geographischen Unterrichtes. F. Deuticke, Leipzig und Wien 1932. - 4. Bösiger K.: Zur Methodik des Geographieunterrichtes an Handelsschulen. "Geographica Helvetica" 1961, H. 3. Kümmerly + Frey, Bern. - 5. BösıGer K.: Vom Wesen und von der Bedeutung des heutigen Geographieunterrichtes. "Korrespondenzblatt" der Geogr.-Ethnologischen Gesellschaft Basel, 8. Jg., 1958, Heft 1 und 2. - 6. Eggersdorfer F.: Jugendbildung (allgemeine Theorie des Schulunterrichtes), München 1930. - 7. Guyer W.: Wie wir lernen. Rentsch-Verlag Zürich 1960. - 8. Huber F.: 
Allgemeine Unterrichtslehre im Abriß, in Klinkarts "Pädagogischen Abrissen". Bad Heilbronn, 6. Auflage, 1959. - 9. Krause K.: Geographische Kausalprofile. Eine Beispielsammlung aus der Praxis für die Praxis. F. Hirt, Breslau 1930. - 10. Merian R. und Nigg W.: Die geographischen Namen im Unterricht. "Geographica Helvetica", 1955, Heft 2. - 11. Meyer E. Gruppenunterricht. Grundlegung und Beispiel. E. Wunderlich, 3. Auflage. Worms 1957. -- 12. Schrass Fr.: Der Erdkundeunterricht. Dürrsche Buchhandlung, Bonn 1953. - 13. Die Neue Schulpraxis. Monatsschrift für zeitgemäßen Unterricht. Zollikofer, St. Gallen. - 14. Wagner P.: Methodik des erdkundlichen Unterrichts, allgemeiner Teil. Quelle und Meyer, Leipzig 1925.

\section{LES EXAMENS ET LES RÉPÉTITIONS DANS L’ENSEIGNEMENT DE LA GÉGRAPHIE DES CLASSES MOYENNES ET SUPÉRIEURES}

Répéter, examiner et mettre des notes sont des activités importantes et fondamentales pour le maître et c'est pour cette raison qu'elles ne doivent pas être abandonnées au hasard.

Dans notre temps de subdivison des branches, l'on doit prévoir - pour arriver à des répétitions fréquentes, méthodiques et continues des sujets importants — suffisamment de temps. Avec la mémorisation d'une quantité rationnelle de noms et de faits, on doit aussi attribuer une grande importance à l'utilisation par l'élève de ce qu'il a appris.

Pour l'enseignement de la géographie, dans les classes moyennes et supérieures, on doit attribuer une importance particulière au développement de l'imagination et de la culture ainsi qu'à la création d'une conception fondamcntale ct claire de la géographie, ceci dans le cadre de la formation générale et des programmes scolaires.

Les répétitions et les examens peuvent, suivant les degrés et le genre d'école, être transformés en exercices stimulants et intéressants, sans que les nombreuses variations possibles conduisent à un jeu sans système. Les points de vue mentionnés et les cxemples rassemblés proviennent de l'enseignement à l'Ecole Cantonale de Commerce de Bâle et sont spécialement destinés à inciter le jeune maître de géographie à faire des travaux d'examen ou bien des répétitions sans notes.

\section{Prof. CHARLES BURKY †}

Am 16. Oktober 1962 starb in seinem 75. Altersjahre, doch unerwartet für Familie, Freunde, Kollegen und Schüler, Charles Burky, Professor für Geographie an der Universität Genf. 1887 in dieser Stadt geboren, hat er ihr als akademischer Lehrer mehr als 5o Jahre gedient und darf daher als ihr eigentlicher «Praeceptor geographiae»gelten. Sein wissenschaftliches Rüstzeug holte er sich auf den Universitäten seiner Vaterstadt, Berlins sowie Leipzigs, wo er 1911 bei Joseph Partsch mit der Dissertation «Die Siedlungen des Rhonequertales in ihrer Abhängigkeit von den Formenelementen des Tales» promovierte. Auch in der Folgezeit wandte er sich mehr und mehr anthropogeographischen Fragen zu, zumal ihm deren Studium als Nachfolger W. Rosiers (seit 1924) an der ökonomisch-sozialen Fakultät der Universität Genf zur Pflicht gemacht wurde. Um die «Géographie humaine» seinen Schülern möglichst lebendig und einprägsam zu vermitteln, schuf er sich eine eigene Methode, die in einer Vorlesungen und Übungen eng verknüpfenden Viergliederung der Darbietung: Theorie, Anwendung, Entwicklung, Fragen der Gegenwart zum Ausdruck kam. Er betonte schon früh, daß die Geographie sich nicht in der Deskription erschöpfen dürfe, sondern als eine normative Wissenschaft betrieben werden müsse, ja im Grunde eine «Philosophie»sei (GH 1957, S. 25t). Mit dieser auf Vorträgen und in Zeitschriften vertretenen Auffassung empfing Burky ein weites Echo nicht nur bei seinen Studenten, sondern auch in internationalen Kreisen. Den deutlichsten Beweis hiefür lieferte die Einladung der UNESCO, Vorträge zu halten, die unter anderem der «Geographie als Faktor des Friedens» eine weltweite Wirkung verschafften. Seine außerordentliche Hilfsbereitschaft fand wohl den leuchtendsten Ausdruck in der französischen Übersetzung des dreibändigen Werkes von J. Früh «Geographie der Schweiz», mit welcher er seinen französisch sprechenden Kollegen die Auffassung der «deutschsprachigen» Geographie, ebensosehr aber auch seinen Mitbürgern eine monumentale Leistung der Erdkunde überhaupt nahebrachte. Mit zunehmendem Alter mehrten sich seine Bindungen an zahlreiche Körperschaften, von denen nur die Mitgliedschaft zur Geographischen Gesellschaft in Genf, zur Schweizerischen Vereinigung für Landesplanung und zum «Haus der europäischen Gemeinden», dessen Direktor er war, genannt seien. Auch diese Kontakte gaben ihm willkommene Gelegenheit, seine Ideen zu verwirklichen und fruchtbar zu machen, wobei er immer wieder auch für Tageszeitungen zur Feder griff. Ein Verzeichnis von mehr als 150 Titeln belegt ebenso seine Schaffenskraft wie seine Geistesfülle, die in einem gleicherweise klaren und bilderreichen Stil Ausprägung empfing. In diesem Zusammenhang sei auch daran erinnert, daß Professor Burky sich als Mitredaktor bei der Herausgabe der zweibändigen Länderkunde «Die Erde» des Verlages Hallwag (1950-57) zur Verfügung stellte, für die er die Erdteildarstellungen sowie eine Reihe von Länderporträts (Nordeuropa, Ozeanien) und die französische Übersetzung beisteuerte. Von seinen Reisen in Nordeuropa und Afrika (Marokko, Sahara, Kanarische Inseln) zeugen zahlreiche Vorträge in seiner Gesellschaft, die er mehrfach präsidierte. $19+9$ hatte er die Freude, die Eidgenossenschaft und die geographischen Gesellschaften der Schweiz am internationalen Geographenkongreß zu vertreten und 1958 die Geographische Gesellschaft Genf zum Fest des Hundertjährigen 